\title{
The Hybrid Technique for Edge Detection using Bio- inspired Techniques
}

\author{
Prachi Bansal \\ Department of Computer Science \& Engineering \\ MIET Meerut, India
}

\author{
Mukesh Rawat \\ Department of Computer Science \& Engineering \\ MIET Meerut, India
}

\begin{abstract}
The image processing is the technique which is applied to process the digital information stored in the form of images. The edge detection is the technique of image processing which detect the points at which the image properties changed at steady rate. In this paper, the bee colony based edge detection technique is proposed which is the enhanced version of the existing edge detection technique based on ant colony optimization. The proposed technique is implemented in MATLAB and it is been analyzed that it performs well in terms of accuracy and execution time.
\end{abstract}

\section{Keywords}

Edge detection, Bee colony Optimization BCO, Ant Colony Optimization (ACO), Artificial Bee colony Optimization (ABC), CUCKOO search (CS) Algorithm, Bee Colony Optimization (BCO)

\section{INTRODUCTION}

Image processing is any form of signal processing for which the input is a digital image, such as a photograph or video structure; the output of digital image processing may be either a digital image or a set of characteristics or parameters related to the image. Most image-processing methods involve treating the image as a 2-D signal and applying standard signalprocessing techniques to it. An edge may be defined as set of linked pixels that forms a border between two divided areas. Edge detection is an essential tool in digital image processing and computer vision in area of feature detection and feature extraction [1]. The main aim of edge detection is to identifying the points in a digital image at which image brightness modifies quickly. Normally edge detection is a process that inputs a grayscale image and then produces a binary image to indicate the edges of objects. The edge representation helps to reduce the quantity of the data to be proceeding but it retains the essential information regarding shape of the objects in the scene [2]. The main possessions of the edge detection method is its capability to abstract the precise edge line with good orientation as well as more literature about edge detection has been available in the past three decades. Edge detection is an essential tool for image segmentation. Edge detection transforms original image into edge images benefits from the changes of grey tones. It is a major process detects and outlines of an object and boundaries among objects and the background in the image.
Edge detection is the most familiar approach for detecting significant discontinuities in intensity values. Optimization techniques are those which give the best fit solution. It is used to solve complex computational solutions. It finds out the best result from the given feasible solutions [4]. There are several techniques which are based upon the natural phenomena.

\subsection{Bee Colony Optimization}

Bee Colony Optimization is nature inspired technique. It is based upon the swarm intelligence technique. It is metaheuristic technique which is concern with memory based searching. Bee colony optimization technique is used to find the best path from the number of the solutions. It is decentralized and self-organizing technique. There are two types of bee which are present in the bee hive. These are in hundred and thousand in numbers which work together. There is one Queen Bee which presents in the bee hive and lay eggs. BCO is an impeccable model of swarm intelligence. The BCO method is a "bottom-up" style to demonstrating where distinct kinds of artificial mediators are created by analogy with bees. Artificial bee agents collaboratively resolve composite combinatorial optimization problem. $\mathrm{BCO}$ is a meta-heuristic procedure which uses the swarm actions of bees and collective intelligence to contract with combinatorial difficulties. Bee Algorithm is used for finding the best imaginable answers for optimization difficulties. Bee algorithm contains of 2 types of alternating pass that donate in a sole step, forward pass and backward pass. Both passes are problem dependent. During forward pass each bee is allocated with a vacant solution. All bees discover search space on separate basis for number of predefined moves. Partial/Complete answers are calculated by every bee. This assessment depends on individual exploration and past involvement. After that bees go back to hive or cluster and start the second stage of first step i.e. backward pass. Throughout this all bees contribute in choice making procedure and all assessed answers are united by every bee by performance waggle dance which is in shape of digit. The answers vary from bee to bee. This is the only time when bees converse with one other and the best among all solution is well-thought-out as partial/complete solution of a problem. Only those resolutions will be loyal that will be giving best results. 


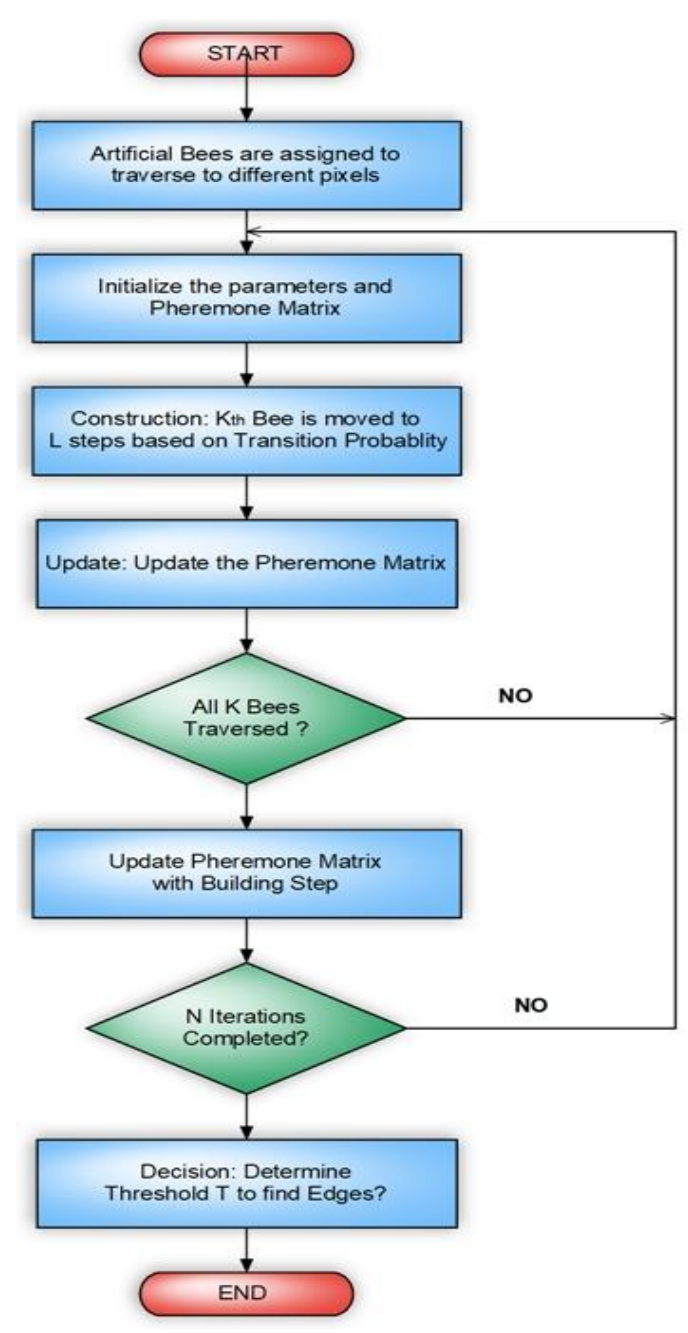

Figure 1: Flow Chart for Bee Colony

\subsection{Ant Colony Optimization}

Ant Colony Optimization is a stream of Artificial Intelligence algorithms that depend on the pretend of the social insects' behavior, and especially ants'. These algorithms use mediators, that are known as "ants", for the enquiry of the best solution of a difficulty, for example the shortest track amid some spaces that might be foodstuff for the colony, just like happening with the true ant colonies.

These mediators or agents, are building through iteration the solutions of the problem. The possibility for an ant to visit a city is affected from the quantity of pheromone that every mediator notices throughout its examination. Pheromone is a matter that ants produce and credit along the trails that have navigated, making them more eye-catching for the next ones that might pass from the same point, while the previously existing pheromone is vanishing as time passes. Throughout the progress of the procedure, the false pheromone is placed after the building of a complete tour-solution on each and every city that was chosen and stayed for the construction of it. In this way, the quantity of pheromone is the heuristic data at a given timing point, imitating the experience of the colony about the possible solutions of the problem.

If the likely solutions are studied as a chart with the ants moving from town sr to $\mathrm{sr}+1$, then the pheromone level that occurs after the passing of a mediator from a possible explanation is given from calculation:

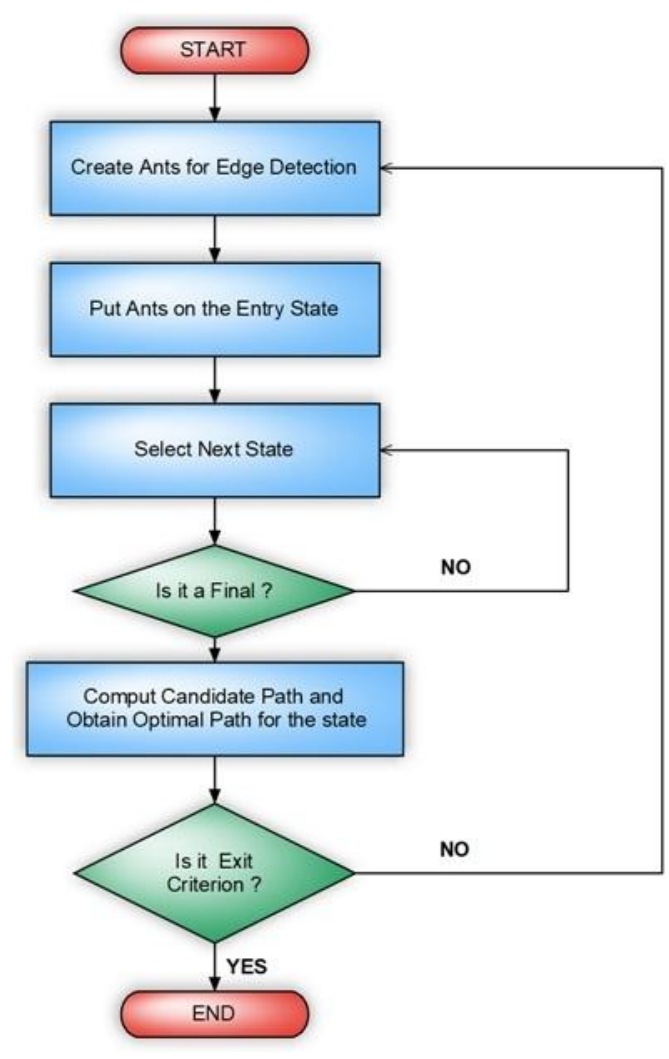

Figure 2: Flow Chart for Ant Colony

\subsection{Cuckoo Search Algorithm}

CUCKOO search (CS) algorithm is put frontward by Yang \& Deb in 2009, which pretends the cuckoo's looking for nest and reproducing activities and announces levy flight mechanism into it, which is able to rapidly and capably find the ideal clarification. This algorithm is primarily based on 2 aspects: the cuckoo's parasitic reproduction mechanism and Levy flights search standard. In nature, cuckoos use an unplanned method or a quasi-random method to pursue bird's nest location. Most of cuckoos lay their eggs in other bird nests and let the host nurture their cubs instead of them. If a host found that the eggs are not its own, it will either throw these unfamiliar eggs away from the nest or abandon its nest and build a new nest somewhere else.

Cuckoo search is one of the optimization algorithms which is used in various fields of image processing. This search carries concept from various cuckoo spices. Earlier this algorithm is used for complex computational problems. Later on this research is applied with PSO and Genetic algorithm and finds out that it achieves better results than PSO and Genetic algorithms. Basically cuckoo search is deal with multimodal problems naturally and efficiently. CS algorithm revolves around the behavior of obligatory brood parasitism of some species of cuckoo as well as the Levy Flights (after the name of French mathematician Paul Pierre Levy) of some birds and fruit flies which follow the random walk of heavy tailed probability distribution step size.

\section{EDGE DETECTION}

Edges in an image are attributed to the sharp transition from high intensity to low intensity regions and provide significant information about an object. A number of methods are in the literature such as the statistical methods, difference methods and curve fitting based methods. Classical methods are based on calculating the first directional derivative in order to 
determine the location of the edges. The zero-crossing edge detectors and the second derivatives incorporated with the Laplacian operator give better accuracy than first derivative operators. Canny edge detector, which is based on the concepts of Gaussian detectors, is one of the most frequently used edge detectors. It gives better performance but is computationally expensive than classical methods. All these methods extract edges by adopting specific formulas and are used with smoothing functions. The major drawback with all the conventional approaches of edge detection comes with the drawback that all these approaches are computationally expensive. The reason lies in the fact that any operation has to be executed for every pixel in the image and thus the computational time gets proportional to the image.

Basic ACO grounded method effectively abstracts the edges of the digital image. Primarily definite number of ants is circulated unsystematically on the digital image. These ants inform their pheromone intensity in each of the iteration. This method uses basic set of rubrics to inform the pheromone intensities.

\section{REVIEW OF LITERATURE}

In this paper [7] that image processing is a technique which used to convert analog image into digital image and helps to improve the quality of the image. Furthermore Nature inspired optimization algorithms are the algorithms which adopts processes running in the nature. These algorithms follow the principle of Charles Darwin of "Survival of Fittest". This paper reveals the detailed explanation of various applications of image processing and how the optimization algorithms work in particular application.

In this paper [8] that an improved ant colony algorithm for image segmentation with a higher efficiency and better edge detection effect. Some crucial improvements include: (a) in the initial stage of segmentation, an idea is brought of fuzzy cluster, enabling the ants to assemble quickly to the edge of the image. (b) In later stage, they introduced an edge search strategy to the edge of the image. A follow-up experiment shows that the improved algorithm is able to segment images in a more efficient manner.

In this paper [9] that a Bee colony optimization and ant colony optimization both are meta-heuristic techniques based on swarm intelligence. Both have no centralized controller and self-organizing techniques. In ACO ants back tracking route to food source using pheromones. In $\mathrm{BCO}$, bees use Path Integration and use direct path to come back to hive instead of back tracking their original route. Bee colony optimization algorithm is more efficient than ant colony optimization algorithm as it takes lesser number of steps when finding and collecting the food.BCO algorithm requires less computation time to complete task so it is more scalable.

In this paper [10] that detected forgery images is one of the challenging task. In this paper first of all classification of image forgery detection technique has been discussed. After that pixel based detection is also defined. A technique for copy-move forgery detections discussed. But this technique is applied on shifted region only. Another technique fast-copymove technique is also defined. Proposed technique is developed to cover all the limitation of the both the techniques and fast copy moving technique. There is no such method that achieved $100 \%$ robustness against post processing operations.

In this paper [11] the technique to remove shift key point attacks from the image for the betterment of SIFT-based copy-move forgery detection. To handle these types of attacks three forensic techniques have discussed in this paper. This is done to remove local and global shift based key points. First of all they verified the technique for the removal of the shift function after that they tried to increase robustness of the technique. They apply the detectors to a practical image forensic scenario of SIFT-based copy-move forgery detection to assume the SIFT-based detectors and as a result give new proposed technique.

In this paper [12], that it is very easy to tamper the images. Image forensic can be used to determine the protection of the image. In this paper they have discussed cope move forgery image problem in which location of one image can be move to another image by hiding its details. So forgery detection is one of the major issues in the image processing. In this paper, they have discussed the various copy move forgery detection techniques which includes Block based \& Key Point based techniques.

\section{BASIC THEORY}

Edges are significant local changes of intensity in an image. And edges typically occur on the boundary between two different regions in an image. An edge in a digital image is a noteworthy native alteration in the digital image concentration, typically related with a cut-off in either the digital image concentration or the first derivative of the digital image concentration. Disjointedness in the digital image intensity can be either (1) step discontinuities, where the digital image concentration sharply changes from one rate on one side of the break to a different value on the conflicting side, or (2) line discontinuities, where the digital image concentration abruptly changes rate but then returns to the initial value within some short distance. However, step and line edges are rare in real digital images. Because of lowfrequency modules or the flattening presented by most sensing strategies, sharp cut-offs rarely exist in real signals. Step edges turn out to be ramp edges and line edges become roof edges, where concentration deviations are not sudden but happen over a fixed distance.

\subsection{Steps in Edge Detection Algorithms}

The 4 steps of edge detection

4.1.1 Smoothing: conquer as much noise as imaginable, without destroying the correct boundaries.

4.1.2 Enhancement: put on a filter to improve the superiority of the edges in the digital image.

4.1.3 Detection: define which edge pixels should be thrown away as noise and which should be reserved (typically, thresholding delivers the standard used for edge detection).

4.1.4 Localization: define the particular position of an edge (sub-pixel steadfastness might be essential for some applications, that is, guess the position of an edge to improve, than the spacing between pixels). Edge dilution and linking are usually compulsory in this stage.

There are several procedures of edge detection, but all of them can be grouped into 2 groups,

\section{(A.) search-based and \\ (B.) zero-crossing based.}

The search-based approaches notice edges by primary computing a amount of edge strength, typically a first-order derivative appearance such as the gradient magnitude, then 
searching for native directional maxima of the gradient magnitude using a calculated approximation of the native orientation of the edge, typically the gradient path. The zerocrossing based approaches exploration for zero crossings in a second-order derivative appearance calculated from the digital image in order to catch edges, typically the zero-crossings of the Laplacian or the zero-crossings of a non-linear disparity expression. As a pre-processing stage to edge detection, a smoothing phase, naturally Gaussian smoothing, is nearly constantly applied.

\section{PROPOSED METHODOLOGY}

In an ant colony optimization algorithm, an ant works as a simple computational agent which brings out a solution for the arising problem. An iteration program keeps executing the problems as a continuous program. The solutions resulting are referred to as solution states which are executed when algorithm runs. A complete intermediate solution is achieved after the steps are executed individually. Feasible expansions are moved from current state to the states that are available in probability. The two listed factors decide the probability of the movement of ant from state to state; first is the heuristic indication the prior of the move which will show the attractiveness of the particular move, and the second which shows the level of move as a trial showing how beneficent it has been to use that move in the past. The trial level will only help in deciding the priority of its use which increases or decreases the level of trails according to their good or bad solutions. At the end all the trials are updated. The probability of ant movement from one state to another is decided by the amount of pheromone. $0 \leq$ parameter is used to control the effects made by the desirability of the state. $\geq 1$ controls the influence and represents the attractiveness and the trail methods of the state. All the trails are updated when the ants have completed all executions. The pheromone evaporation coefficient indicates the amount of pheromone deposited by the transition state as well as an ant. It is typically given by a TSP problem, in which the cost of the ant's tour is constant.

\section{EXPERIMENTAL RESULTS}

The proposed algorithm has been implemented in MATLAB and the results are analysed in terms of accuracy, execution time, Image Pixel Range.

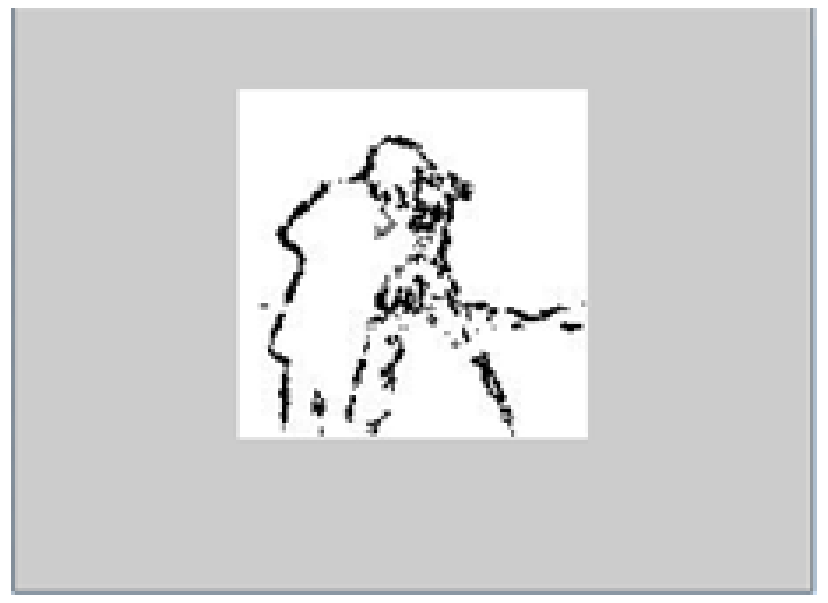

Figure 3: Edges Detected by Artificial Bee Colony

As shown in Figure 3, the edges created with the help of existing Artificial Bee Colony Method, are not connected well and the little parts in the image are ignored. The total execution is more than proposed method.

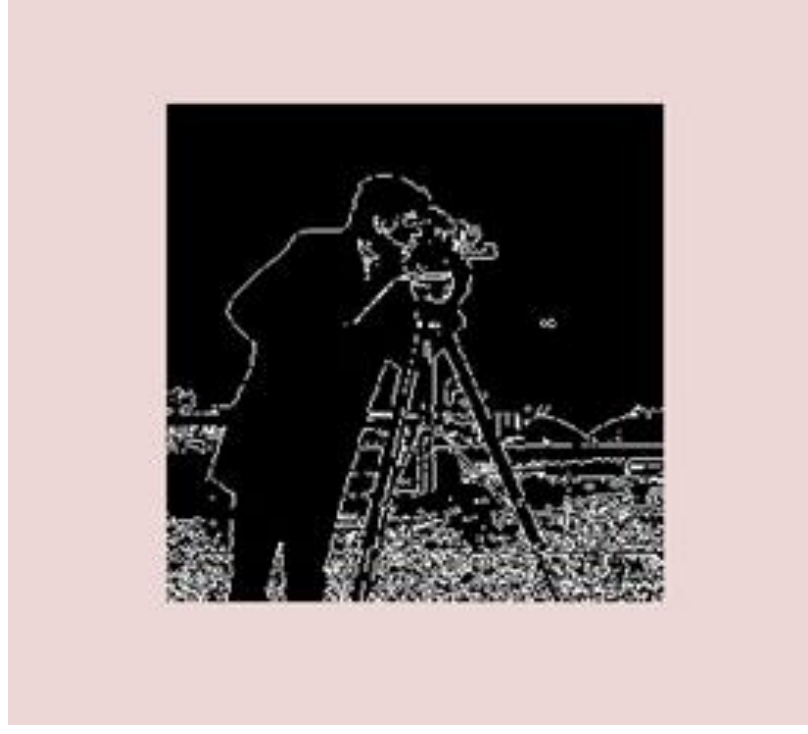

Figure 4: Edges Detected by Proposed Method

Considering Figure 4, we can see a huge improvement in continuity of edges and every little part in the image is bounded by an edge. The edges are sharp and clear and the total execution time is more than less than Artificial Bee Colony Method.

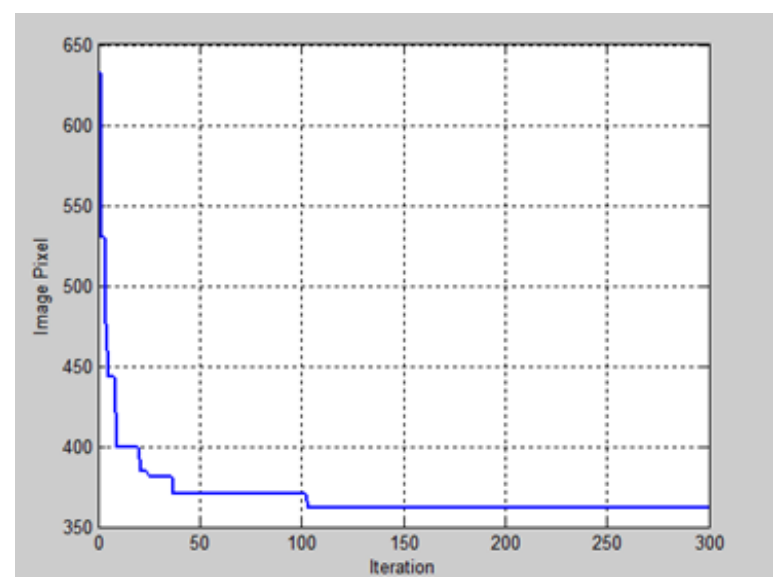

Figure 5: Graph plotted between Image Pixel and Iteration of Artificial Bee Colony

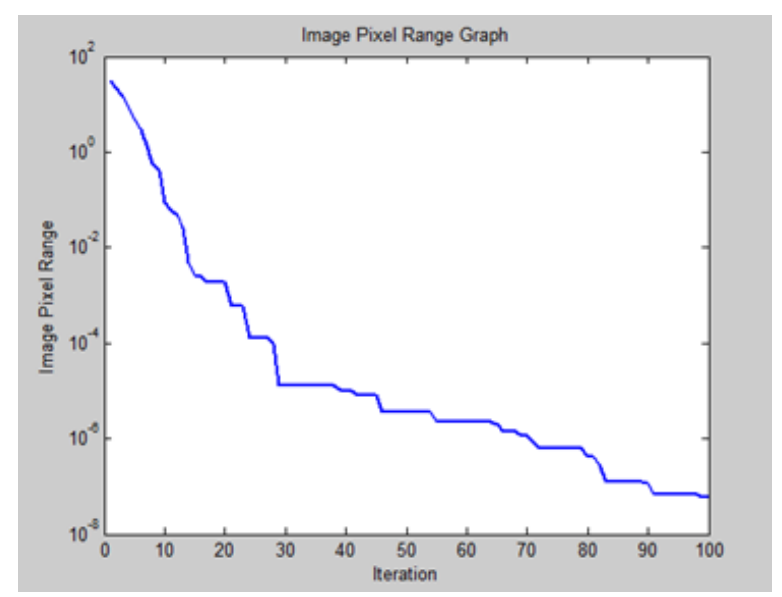

Figure 6: Graph plotted between Image Pixel and Iteration of Proposed Method 


\subsection{Result}

As it can be seen in the Table 1 shown below that there is a big variation between accuracy level of existing algorithm and proposed algorithm. As the accuracy is more important than execution, and with this proposed algorithm, it has been achieved. So it can be said that the proposed modification in the existing Bee Colony Algorithm will give a huge improvement to the edge detection techniques.

Table 1: Comparison with Existing Algorithm, between Execution Time and Accuracy

\begin{tabular}{|c|c|c|}
\hline Parameters & Existing Algorithm & $\begin{array}{c}\text { Proposed } \\
\text { Algorithm }\end{array}$ \\
\hline Execution Time & 146.2556 & 50.250413 \\
\hline Accuracy & 76.335 & 91.808 \\
\hline
\end{tabular}

Table 2: Comparison among diff. parameters of Algorithm

\begin{tabular}{|c|c|c|}
\hline PARAMETERS & $\begin{array}{c}\text { Artificial Bee } \\
\text { Colony }\end{array}$ & $\begin{array}{c}\text { Proposed } \\
\text { Method }\end{array}$ \\
\hline PSNR & 18.104 & 60.161 \\
\hline MSE & 1013.925 & 84.852 \\
\hline RMSE & 31.842 & 9.211 \\
\hline $\begin{array}{c}\text { FAULT DETECTION } \\
\text { RATE }\end{array}$ & 0.82658 & 0.246 \\
\hline $\begin{array}{c}\text { PERFORMANCE } \\
\text { (ACCURACY) }\end{array}$ & 76.335 & 91.808 \\
\hline
\end{tabular}

\section{CONCLUSION}

Considering the research papers studied, it can be said that significant division of the study was focused towards modifying and hybridizing $\mathrm{ABC}$ to resolve varied set of troubles, counting those on data clustering, designing problems, medical image processing, scheduling problem etc. The greater part of applications developed or proposed were intended at resolving constrained and unconstrained optimization troubles.

The $\mathrm{ABC}$ has come to be known as a influential and strong global optimization algorithm, able of tackling uni-modal and multimodal, non-differentiable, nonlinear objective functions. Although the motivating force of the $\mathrm{ABC}$ is its neighbor operators, several attempts have been made by researchers in several disciplines to increase the efficiency and performance of algorithm by introducing new operators. Still, there still reside many new areas of application and open troubles in which $\mathrm{ABC}$ could be adopted. In conclusion, $\mathrm{ABC}$ stays as a promising and interesting algorithm, which would continue to be extensively used by researchers across several fields. Its potential advantage of being easily hybridized with different meta-heuristic algorithms and elements make it robustly viable for sustained consumption for more exploration and modifications possibilities in many more years is yet to come.

\section{REFERENCES}

[1] Preeti Yadav, YogeshRathore, Aarti Yadav," DWT Based CopyMove Image Forgery Detection",
International Journal of Advanced Research in Computer Science an Electronics Engineering Volume 1, Issue 5, July 2012

[2] S.Murali, Govindraj B. Chittapur ,Prabhakara H. S and Basavaraj S. Anami, "Comparison and Analysis of Photo Image Forgery Detection Techniques", International Journal on Computational Sciences \& Applications (IJCSA) Vo2, No.6, December 2012

[3] Ms. P. G.Gomase, Ms. N. R. Wankhade, " Advanced Digital Image Forgery Detection- A Review", IOSR Journal of Computer Science (IOSR-JCE) e-ISSN: 22780661, p-ISSN: 2278-8727 PP 80-83 2014

[4] Andrea Costanzo, Irene Amerini, Roberto Caldelli, Mauro Barni, "Forensic Analysis of SIFT Keypoint Removal and Injection", IEEE TRANSACTIONS ON INFORMATION FORENSICS AND SECURITY, VOL. 9, NO. 9, SEPTEMBER 2014

[5] S. Xiaoya and L. Yan, "Improved Artificial Bee Colony Algorithm for Assignment Problem", Microelectronics \& Computer, vol. 29, no. 1, 2012

[6] W. Hai-ning and S. Shou-qian, "Adaptive Ant Colony Optimization Algorithm Based on Maturity", Microelectronics \& Computer, vol. 27, no.8, pp. 140144,2010

[7] Manish Dixit, Sanjay Silakari and Nikita Upadhayay, "Nature Inspired Optimization Algorithms: An Insight to Image Processing Applications", International Journal of Emerging Research in Management \& Technology, Volume-4, Issue-5, 2015

[8] Xumin Liu, Xiaojun Wang, Na Shi and Cailing Li, "Image Segmentation Algorithm Based on Improved Ant Colony Algorithm", International Journal of Signal Processing, Image Processing and Pattern Recognition Vol.7, No.3, pp.433-442, 2014

[9] Mandeep Kaur Bedi, Sheena Singh, "Comparative Study of Two Natural Phenomena Based Optimization Techniques", International Journal of Scientific \& Engineering Research Volume 4, Issue3, March, 2013

[10] Ms. P. G.Gomase, Ms. N. R. Wankhade, "Advanced Digital Image Forgery Detection- A Review", IOSR Journal of Computer Science (IOSR-JCE) e-ISSN: 22780661, p-ISSN: 2278-8727 PP 80-83 2014

[11] Andrea Costanzo, Irene Amerini, Roberto Caldelli, Mauro Barni, "Forensic Analysis of SIFT Keypoint Removal and Injection", IEEE TRANSACTIONS ON INFORMATION FORENSICS AND SECURITY, VOL. 9, NO. 9, SEPTEMBER 2014.

[12] Gagandeep Kaur, Manoj Kumar, "Study of various copy move forgery attack detection in digital images", INTERNATIONAL JOURNAL OF RESEARCH IN COMPUTER APPLICATIONS AND ROBOTICS, Vol.3 Issue 9, Pg.: 30-34 September 2015. 